The wide range of reactivity of vitamin $B_{6}$ with a number of drugs and other chemicals encountered in the environment must be considered in the diagnosis. These interactions involve a condensation between the xenobiotic, and pyridoxal phosphate, and vitamin $B_{6}$ absorption, coenzymes, and specific vitamin $\mathrm{B}_{6}$-dependent enzymes can also be affected. Isoniazide is probably the most extensively studied drug with respect to effects on vitamin $B_{6}$ metabolism. Other drugs reported to interact with vitamin $\mathrm{B}_{6}$ include ampicillin, anticonvulsants, levodopa, oral contraceptives and ethanol. The mechanism of anticonvulsant drug and vitamin $\mathrm{B}_{6}$ interaction is not well determined. One study showed that the vitamin was decreased only in males treated with anticonvulsants. Another showed that pyridoxine supplements resulted in lower plasma phenytoin and phenobarbital concentrations. An interaction between the vitamin and dyes added to medications during manufacture has also been demonstrated (Dubick MA. Interactions of vitamin $\mathrm{B}_{6}$ (pyridoxine) and xenobibotics. In Nutritional Toxicology, Vol III, ed. JN Hathcock. New York, Academic Press, 1989.)

\title{
DISCONTINUING MEDICATION: RECURRENCE RISK FACTORS
}

The results of antiepileptic drug discontinuation in epileptic children who were seizure free for at least 2 years are reported from the Services of Child Neurology and Psychology, Department of Neurology, Clinics Hospital University of Sao Paulo Medical School, Sao Paulo, Brazil. Twenty children (28\%) had a recurrence, $75 \%$ during discontinuation or less than 6 months after discontinuation of AED. The risk factors related to seizure recurrence were 1) more than 10 seizures before seizure control, 2) abnormal EEG in the year before discontinuation, 3) focal neurologic signs and/or mental retardation, and 4) a mixed seizure pattern. Two or more risk factors were associated with recurrence of seizures in 14 children $(70 \%)$, whereas patients with none or only 1 risk factor had no recurrence $(72 \%)$. (Gherpelli JLD et al. Discontinuing medication in epileptic children: a study of risk factors related to recurrence. Epilepsia July/Aug 1992; $\underline{33}: 681-686$.) (Reprints: Dr. J.L.D. Gherpelli, Service of Child Neurology, Department of Neurology, Hospital das Clinicas da F.M.U.S.P., Sao Paulo 01000, P.O. Box 8091, Brazil.)

COMMENT. Risk factors may be of help in determining the safety of discontinuing antiepileptic medications in children who are seizure free for a period of at least 2 years. There was no correlation between age at seizure onset and recurrence in this study, which is at variance with some other reports.

A finding of a higher sleep tendency in children with epilepsy than in controls, even 4-5 months after drug discontinuation, was unexpected in a study from the University of Lund, Sweden (Palm L et al. Epilepsia July/Aug 1992; 33:687-691). A long time had passed since the last reported seizure and the EEG was free of epilepti-form activity in most cases, so that the sleepiness could not be a direct effect of epileptic seizures. 